\title{
Resilient Cyber-Physical Energy Systems using Prior Information based on Gaussian Process
}

\author{
Charalambos Konstantinou, Senior Member, IEEE, Olugbenga Moses Anubi, Senior Member, IEEE
}

\begin{abstract}
The power grid infrastructure is a large-scale, heterogeneous, and complex cyber-physical system which forms the lifeline of modern societies. The trend of tight coupling of physics, communication and computation in cyber-physical energy systems (CPES) is evident by the inclusion of numerous measurement sensors. This contributes to enhancing the monitoring and control functionalities of CPES. At the same time, the occurrence of adverse effects constitutes a vital dimension of CPES operation. Increasing the resilience of critical energy systems of key importance for safeguarding the national economy and security. This paper considers the problem of optimal estimation with sensing measurements subject to arbitrary corruption resulting from adverse effects. Such signals can cause false situation awareness and/or trigger a sequence of cascading effects leading to an ultimate system failure. We formulate the problem as a constrained optimization with additional prior information posed as a set inclusion constraint on the measurement vector. It is shown that if the prior set satisfies certain conditions, the resulting recovery error bound is improved. The approach demonstrates enhancement of the CPES resiliency by using the Gaussian process as the basis of a prior generative probabilistic regression model using historical data. The validation of the resiliency mechanism using prior information is performed using the New York Independent System Operator (NYISO) grid data, demonstrating $100 \%$ successful state recovery for up to $60 \%$ of CPES sensor failures.
\end{abstract}

Index Terms-Cyber-physical energy systems, resiliency, adverse cyber-physical events, prior information.

\section{INTRODUCTION}

Cyber-physical systems (CPS) refer to a generation of systems with tightly-integrated communication, computational and physical capabilities that can interact with humans through many new modalities [1]. Such systems are fundamental to the operation of various safety-critical applications (e.g., smart grid, connected \& autonomous vehicles (CAV), etc). Their failure can cause irreversible damage to the underlying physical system as well as to the humans who operate it or depend on it. The control of CPS is enabled by the proliferation of sensing devices which allow geographically isolated physical plants to be remotely monitored. The measured data are sent via supervisory control and data acquisition (SCADA) systems to central master stations. At the central site, the information is utilized to carry out necessary analysis and control, e.g., determine if a leak has occurred and the level of criticality.

C. Konstantinou is with the Computer, Electrical and Mathematical Sciences and Engineering (CEMSE) Division, King Abdullah University of Science and Technology (KAUST), Thuwal 23955-6900, Saudi Arabia (e-mail: charalambos.konstantinou@kaust.edu.sa).

O. Anubi is with Florida State University, Tallahassee, FL 32310 USA (e-mail: oanubi@fsu.edu).
The transformation of critical infrastructure into CPS is accompanied, besides the operational performance and economic benefits, with important technical challenges [2]. For example, from the physical system perspective the current distribution power system is based on overhead lines and radial feeders that not only are easily damaged by natural disasters such as hurricanes, but also create disasters like the wildfires in California. Resiliency is becoming critical in the context of cyber-physical energy systems (CPES) [3], [4]. Adverse cyberphysical effects (ACEs) including natural disasters such as hurricanes, earthquakes, wildfires, 'silent errors' due to components and manufacturing variability failures, hardware or software faults of smart monitoring devices due to bugs in the code (e.g., operating system, compilers, libraries, etc.), natural effects such as bit flips induced by hardware failures, drive failures, cosmic rays, cyberattacks, or even faults involving the infrastructure design and implementation, will more drastically affect the results of CPS algorithms, and subsequently the operations of systems deployed in critical infrastructures.

Despite decentralized and distributed schemes in large-scale infrastructures, existing diagnosis approaches rely on concepts in which state data are gathered and processed centrally [5], [6]. Their computational efficiency is typically very low and ACEs cannot be handled properly [7]. Moreover, such solutions extend further the threat landscape to include the potential of ACEs at the distributed agent (e.g., false data injection attacks in distributed state estimation could yield an erroneous estimate which could even cause misinformation on the location of outages [8]). Such events can lead to escalating instability in system dynamics. The importance of addressing resiliency is evident by the efforts of various governments agencies [9]. Towards addressing ACEs issues in CPES, thus ensuring reliable operation of the system, the state estimates are utilized for informed decision making of the underlying physical system (e.g, contingency analysis, automatic generation control, etc.) via preventive and corrective actions.

In the literature, resilient state estimation has attracted significant attention [10]. While there are numerous works on the topic, most of them focus on attack-resilient algorithms that consider measurement noise [11], [12], time varying attack support [13], robustness considerations [14] and distributed case [15]. There are also numerous applications including power systems [16], UAVs [17], energy delivery systems [18], autonomous vehicles and networked systems. In contrast with work that utilizes prior information for resilient estimation [19], [20], our work, despite assuming the knowledge of location of ACEs nodes in the topology of the CPES does not assume any information acquired from such nodes. The 
prior information in our propositions can recover system observability, enhancing CPES resiliency against generic ACEs.

In this paper, we propose an estimation algorithm for sustained proper operation of CPES under ACEs by using auxiliary prior information (in our case Gaussian process regression (GPR) market data) to boost CPES operational estimation resiliency. The main advantage of our concurrent model-based and data-driven algorithm is that the resulting synergy of the generalization properties of the model-based method and the accuracy of the data-driven method creates an additional layer of redundancy which can reveal the truth even if portions of the measurement is subject to ACEs. Specifically, we build on our previous works on enhancing the recoverability of resilient estimators by incorporating prior information, either in form of attack-support estimation [21] or through a more general set inclusion constraint [22]. We provide theoretical guarantees of how certain boundedness properties of the prior information set can improve the reconstruction error bound of the resulting resilient estimator. Unlike previous work [13], [23], [24] which depends on the restricted isometry property (RIP) [25], we developed our results by leveraging the latent information contained in the auxiliary model. Moreover, the developed estimator is applied on realistic data acquired from the New York Independent System Operator (NYISO) transmission grid. The prior information generates likelihood-level ellipsoid constraints on the "true" measurement vector via GPR mean and covariance functions of the locational marginal pricing (LMP) of system buses. The simulation experiments demonstrate tremendous improvement in resiliency by using readily available auxiliary measurements to corroborate the estimation process using the proposed scheme.

The rest of the paper is organized as follows: in Section [I] we present the model of the CPES utilized in our work and how GPR is used to encode the prior information of CPES. Section III develops the proposed resilient estimation algorithm against ACEs. Simulation results are reported in Section IV Concluding remarks are provided in Section $\mathrm{V}$ and notation is shown in the Appendix.

\section{Model Development}

In this section, we provide the model details of the CPES used in this work. The physical system considered is the electrical power transmission system of NYISO. The system is mapped to the IEEE 14-bus to facilitate dynamic model development. Furthermore, the cyber model uses the overlaying economic model on the power system, seeking to model the correlation between energy price and voltage measurements.

\section{A. System Model}

The power system model used in our work is based on the IEEE 14-bus benchmark which consists of 14 buses, 5 generators, 11 loads, 3 transformers, and 22 branches as presented in Fig. 1. The state variables are represented by the voltage angles and magnitudes of the system. Measurement sensors are assumed to be placed in all system nodes providing active and reactive power injection and flow. In order to map the bus model with realistic data, 5-minute resolution load data

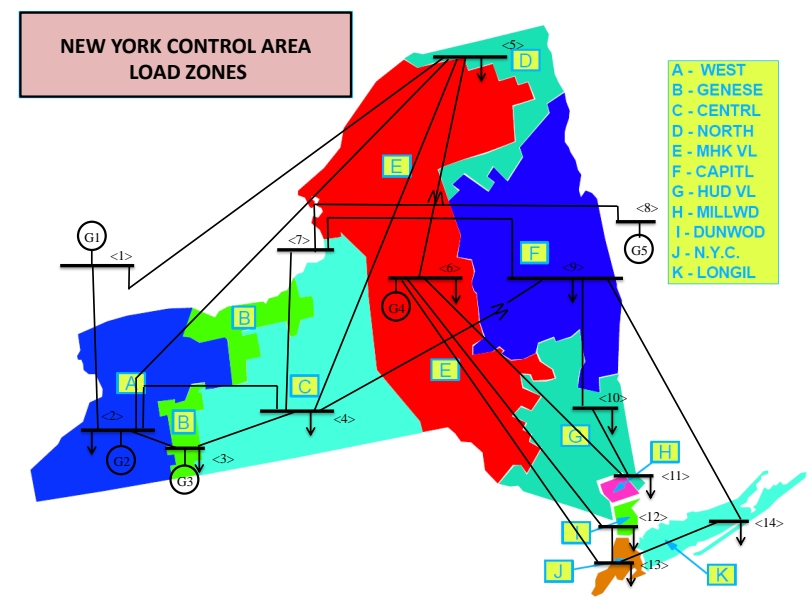

Fig. 1: New York Independent System Operator (NYISO) control area load zones mapped to IEEE 14-bus system.

was obtained from the 11 load zones that exist in NYISO, shown in Fig. 1 [26]. These load zones are mapped to every load bus in the IEEE-14 test system in an ascending order, similarly to process described in [22]. In addition, NYISO load information from each zone is normalized using a constant power factor to the initial active/reactive power of the IEEE 14-bus loads. The ratio of the derived total power to the initial power is used as an input for the 5 generators of the case model to increase their output power [27]. Based on this mapping process, the states of the system $\mathrm{x} \in \mathbb{R}^{n}$ are determined using the nonlinear power flow Newton-Raphson method. Finally, sensor measurements $\mathbf{y} \in \mathbb{R}^{m}, \mathbf{y}=h(\mathbf{x})$ from the system nodes/buses can be computed according to the Jacobian matrix $J_{h}$ of $h(\mathbf{x})$ (for every 5-minute interval) in order to be utilized in our case study. $J_{h}$ represents how each measurement point depends on state variables, i.e., $h: \mathbb{R}^{n} \mapsto \mathbb{R}^{m}$ represents the connection of $\mathbf{x}$ and $\mathbf{y}$ based on the system power flow.

Adverse Cyber-physical Events (ACEs): From the system model, here we summarize the threat model of the ACEs in resilient estimation process. Resiliency, for the purpose of this paper, refers to the capacity of a system to retain functionality while facing disruptions caused by specified ACEs. These disruptions constitute the threat model in question. Specifically, for state estimators, resiliency refers to the tendency to maintain low estimation error when portions of the system measurement is unavailable due to ACEs.

(a) ACEs such as natural disasters, hardware or software faults, cyberattacks, or even infrastructure design and implementation faults, are assumed to compromise $(m-\mathcal{T})>$ $(m-n)$ measurements points from the nodes/buses of the system, where $\mathcal{T} \subset\{1,2, \ldots, m\}$ is the set of the indices of the available (or safe from ACEs) measurements. For example, in the scenario of a stealthy attack, an adversary can compromise communication networks (e.g. IEEE C37.118 communication protocol does not have necessary security mechanisms [28]) and falsify the measurement data directly. Note that, as power systems become intelligent CPES, a set of missing data might not have tremendous impact on the operations of the systems since the CPES could correct 
trivial mistakes or faults. Nevertheless, in the event of false measurements in a consecutive manner received or even if data are not transferred at all to the control center, the CPES might not be able to auto-correct dependent functions; consequently, resulting in system failures [29]. Finally, we do not assume a priori what specific data can be compromised/missing or not, however, we do assume the knowledge of the set of failed/misbehaved sensors.

(b) In a typical CPES, ACEs can affect "either the analog stimulus that excites the sensor, the analog output of the sensors or the digital output of the sensor system that is fed into the control estimation system" [30]. This paper focuses on all sensor-related adverse events, with the premise that such ACEs are improbable to take over (or have full access to) the SCADA systems or the control center. Once ACEs can affect the control center or the SCADA systems, then the system operation is compromised despite having in place the most resilient estimation mechanisms.

(c) Since we focus on leveraging auxiliary prior from a GPR model mapping LMP prices to preserve observability, this paper assumes the current electricity pricing has not been affected by prior ACEs. The regulation of the electricity market and marginal prices are assumed to follow the system marginal costs produced by the real-time dispatch and commitment programs. The LMP at bus $j$ can be written as:

$$
\gamma_{j}=\lambda^{R}+\gamma_{j}^{L}+\gamma_{j}^{C}
$$

where $\gamma_{j}$ is the LMP at bus $j$ in $\$$ MWh, $\lambda^{R}$ the system marginal price at the reference bus, $\gamma_{j}^{L}$ is the marginal losses component of the LMP at bus $j$ which is the marginal cost of losses at bus $j$ relative to the reference bus, and $\gamma_{j}^{C}$ is the congestion component of the LMP at $j$ which is the marginal cost of congestion at bus $j$ relative to the reference bus [31].

\section{B. Prior Model}

In addition to the measurement vectors acquired from buses/nodes via field sensors, our system includes auxiliary models (examples include LMP, locational ambient conditions, transformer ratings, etc.). In our study, auxiliary models of prior market information (LMP) are able to provide an added resiliency layer to the system, i.e., an added layer of redundancy for boosting system resiliency to arbitrary data corruption due to ACEs. We use such market data to build a probabilistic regression GPR model via mapping them to the nodes measurements. Specifically, our GPR model maps LMP prices to bus voltages and angle measurements. In our developed test model of the IEEE 14-bus system with NYISO data, we use for the respective nodes system the LMP information provided by NYISO. LMP data generated at each security constrained economic dispatch (SCED) cycle represents the cost to serve the next increment of load at a node/bus. The GPR model schematic is shown in Fig. 2 .

Data Processing for GPR: The resiliency of CPES against ACEs is enhanced via integrating concurrent model-based and data-driven techniques in order to secure tight thresholds. Based on the system and prior models information, a datadriven scheme based on Gaussian processes (GP) is trained. The selection of GPs is motivated by:

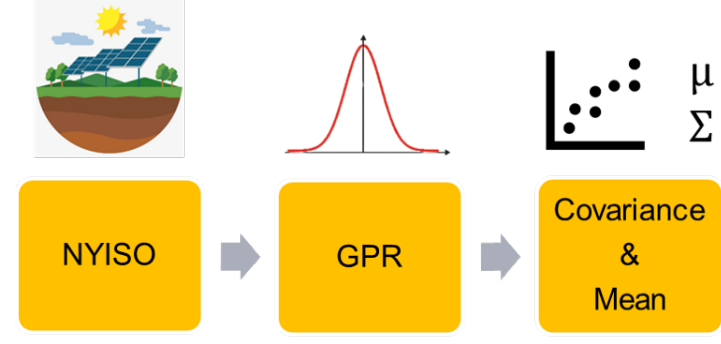

Fig. 2: Gaussian Process Regression (GPR) Model Schematic.

- GP's expressiveness in terms of prediction and uncertainty quantification,

- The final mathematical expression can be integrated with traditional estimation with little efforts, and

- The provision for incorporating prior (possibly knowledge-based) information and the non-dependence on explicit model parameters.

- The training of the GPR model can be adapted easily for recursive online updates as new data points become available.

One drawback of algorithms based on GPs is the resulting computational explosion on large datasets. Fortunately, there is a host of efforts and important results on sparsification of dataset geared towards approximating large datasets with smaller ones while maintaining critical latent information [32].

Remark 1. GPR uses GPs to encode prior distributions over functions. The priors are then updated to form posterior distributions with updated data. Consider a dataset $\mathcal{D}_{\mathcal{R}}=\{\mathbf{Z}, \mathbf{Y}\}: \mathbf{Z} \in \mathbb{R}^{p \times N}$ contains auxiliary variables and $\mathbf{Y} \in \mathbb{R}^{m \times N}$ are output measurements. The goal is to learn $f: \mathbf{R}^{p} \mapsto \mathbf{R}^{m}$ for which $\mathbf{y}_{j}=f\left(\mathbf{z}_{j}\right)+\varepsilon, i=j, . ., N$, $\varepsilon \sim \mathcal{N}\left(0, \operatorname{diag}\left(\sigma_{1}^{2}, . ., \sigma_{m}^{2}\right)\right)$. The problem is ill-defined because there are many functions that explain the data exactly notwithstanding the measurement noise. As a means of regularization, the class of functions for consideration is refined by the restriction $f(\mathbf{z}) \sim \mathcal{G P}\left(\mu(\mathbf{z}), k\left(\mathbf{z}, \mathbf{z}^{\prime}\right)\right)$ to a GP specified by its mean $\mu(\mathbf{z})$ and covariance $k\left(\mathbf{z}, \mathbf{z}^{\prime}\right)$. The covariance can then be specified apriori without an explicit probability distribution. This is where the prior information is encoded in GP. One function commonly used is $V \exp \left(-1 / 2 * l\left\|\mathbf{z}-\mathbf{z}^{\prime}\right\|^{2}\right)$ where hyperparameters $V$ and $l$ implicitly define a smoothnesspromoting prior [22].

Remark 2. Given a query point $\mathbf{z} \in \mathbb{R}^{p}$ for an auxiliary variable, the posterior distribution for the $\Omega_{j}$ node sensor is $p\left(y_{j} \mid \mathbf{z}, \mathcal{D}\right)=\mathcal{N}\left(\mu_{j}(\mathbf{z}), \Sigma_{j}(\mathbf{z})\right): \mu_{j}(\mathbf{z})=$ $\mathbf{k}(\mathbf{z})^{\top}\left(K+\sigma_{j}^{2} I\right)^{-1} \mathbf{Y}_{j}^{\top}$, and $\Sigma_{j}(\mathbf{z})=k(\mathbf{z}, \mathbf{z})-\mathbf{k}(\mathbf{z})^{\top}(K+$ $\left.\sigma_{j}^{2} I\right)^{-1} \mathbf{k}(\mathbf{z}), \quad j=1, . ., m$, where $K \in \mathbb{R}^{N \times N}$ entries are $K_{i j}=k\left(\mathbf{z}_{i}, \mathbf{z}_{j}\right)$ and $\mathbf{k}(\mathbf{z}) \in \mathbb{R}^{N}$ is a vector with entries $\mathbf{k}(\mathbf{z})_{i}=k\left(\mathbf{z}, \mathbf{z}_{i}\right)$. Thus, the overall measurements values posterior distribution is given by: $p\left(\mathbf{y} \mid \mathbf{z}, \mathcal{D}_{\mathcal{R}}\right)=$ $\prod_{j=1}^{q} \mathcal{N}\left(\mu_{j}(\mathbf{z}), \Sigma_{j}(\mathbf{z})\right)$.

Fig. 3 shows the mean relative absolute errors for the GPR model trained and evaluated on actual 5-minute interval load and market data from the NYISO. The raw data is divided into 


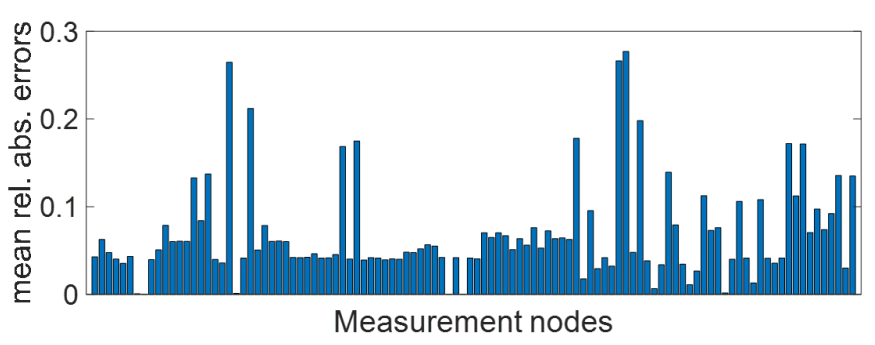

Fig. 3: GPR mapping market data to voltage measurements: relative absolute error performance on NYISO transmission system data.

two portions, with $30 \%$ randomly reserved for validation. The GPR model is trained on the remaining $70 \%$ of the data using the Statistical and Machine Learning Toolbox in MATLAB. The squared exponential kernel was used, with the characteristic length scale and signal standard deviation hyperparameters tuned by the underlying hyperparameter optimization routine implemented in the toolbox. We trained 108 GPR models, one for each sensor node, mapping the auxiliary variable to the corresponding nodal measurement. During the evaluation phase, each GPR model is run on the entire validation set and the mean value of the absolute relative error is computed and reported in the figure. From the figure, it is seen that the model is able to achieve less than $10 \%$ relative absolute error for most measurement nodes and less than $30 \%$ for all measurement nodes. This is expectedly suggestive of a strong correlation between the overlaying market operation and actual physical measurements. Such auxiliary information has significant potential for boosting system resiliency to extreme ACEs if used properly.

\section{RESILIENT ESTIMATION}

In this section, we develop the proposed resilient system awareness algorithm leveraging the auxiliary prior information to preserve observability. The system awareness problem is formulated as a state estimation routine of CPES. Since the focus of this paper is not failure detection and localization, we assume that the failed sensors due to ACEs are identifiable. Consider the observation model:

$$
\mathbf{y}=H \mathbf{x}+\varepsilon,
$$

where $H \in \mathbb{R}^{m \times n}, \quad n \ll m$ is a locally linearized system model above mapping from some internal states $\mathbf{x} \in \mathbb{R}^{n}$ to a vector of observation $\mathbf{y} \in \mathbb{R}^{m}$, with measurement noise $\varepsilon \in \mathbb{R}^{m}$ which is assumed to be bounded as $\|\varepsilon\|_{2} \leq \delta$ for a known upper bound $\delta>0$. Let $\mathcal{T} \subset\{1,2, \ldots, m\}$ be a set of the indices of the available (or safe from ACEs) measurements. Then the estimation model above reduces to

$$
\mathbf{y}_{\mathcal{T}}=H_{\mathcal{T}} \mathbf{x}+\varepsilon_{\mathcal{T}},
$$

where $H_{\mathcal{T}} \in \mathbb{R}^{|\mathcal{T}| \times n}$ is the matrix obtained from $H$ by extracting only the rows corresponding to the indices in $\mathcal{T}$. Thus, the least-square estimator (or decoder) is of the form

$$
\hat{\mathbf{x}}_{\mathcal{T}}=\mathcal{D}\left(\mathbf{y}_{\mathcal{T}}\right) \triangleq \underset{\mathbf{x}}{\arg \min }\left\|\mathbf{y}_{\mathcal{T}}-H_{\mathcal{T}} \mathbf{x}\right\|_{2} .
$$

Let $\mathrm{x}^{*} \in \mathbb{R}^{n}$ be the unknown true state of the system that we desire to estimate. If $|\mathcal{T}|<n$, then the above estimation problem degenerates and there is no unique solution. Indeed, there is a whole class of valid estimates:

$$
\hat{\mathbf{x}}_{\mathcal{T}}=\mathbf{x}^{*}+N_{\mathcal{T}} \mathbf{z}, \quad \forall \mathbf{z} \in \mathbb{R}^{n-|\mathcal{T}|}
$$

where $N_{\mathcal{T}} \in \mathbb{R}^{n \times(n-|\mathcal{T}|)}$ is a matrix whose columns form a basis for the null space of $H_{\mathcal{T}}$. Consequently, the estimation error is potentially unbounded, i.e.,

$$
\left\|\hat{\mathbf{x}}_{\mathcal{T}}-\mathbf{x}^{*}\right\|_{2}=\|\mathbf{z}\|_{2}
$$

is unbounded since $\|\mathbf{z}\|_{2}$ is unbounded. To mitigate the problem with unboundedness, one could regularize the problem as follows:

$$
\hat{\mathbf{x}}_{\mathcal{T}}=\mathcal{D}\left(\mathbf{y}_{\mathcal{T}} ; \lambda\right) \triangleq \underset{\mathbf{x}}{\arg \min }\left\|\mathbf{y}_{\mathcal{T}}-H_{\mathcal{T}} \mathbf{x}\right\|_{2}^{2}+\lambda\|\mathbf{x}\|_{2}^{2},
$$

where $\lambda>0$ is a regularization parameter.

The following proposition summarises the achievable error bound for the regularized decoder above.

Proposition 1. The decoder $\hat{\mathbf{x}}_{\mathcal{T}}=\mathcal{D}\left(\mathbf{y}_{\mathcal{T}} ; \lambda\right)$ satisfies the error bound

$$
\frac{\lambda}{\lambda+\bar{\sigma}_{\mathcal{T}}} \leq \frac{\left\|\hat{\mathbf{x}}_{\mathcal{T}}-\mathbf{x}^{*}\right\|_{2}}{\left\|\mathbf{x}^{*}\right\|_{2}} \leq 1
$$

where $\bar{\sigma}_{\mathcal{T}}$ is the largest singular value of $H_{\mathcal{T}}$.

Proof. The corresponding solution to the regularized problem is

$$
\hat{\mathbf{x}}_{\mathcal{T}}=\left(H_{\mathcal{T}}^{\top} H_{\mathcal{T}}+\lambda I\right)^{-1} H_{\mathcal{T}}^{\top} \mathbf{y}_{\mathcal{T}}
$$

Thus the associated estimation error is given by:

$$
\begin{aligned}
\left\|\mathbf{x}^{*}-\hat{\mathbf{x}}_{\mathcal{T}}\right\|_{2} & =\left\|\left(I-\left(H_{\mathcal{T}}^{\top} H_{\mathcal{T}}+\lambda I\right)^{-1} H_{\mathcal{T}}^{\top} H_{\mathcal{T}}\right) \mathbf{x}^{*}\right\|_{2} \\
& =\left\|\left(I-(\Sigma+\lambda I)^{-1} \Sigma\right) U_{\mathcal{T}}^{\top} \mathbf{x}^{*}\right\|_{2},
\end{aligned}
$$

where $H_{\mathcal{T}}$ admits the singular value decomposition $H_{\mathcal{T}}^{\top} H_{\mathcal{T}}=$ $U_{\mathcal{T}} \Sigma U_{\mathcal{T}}^{\top}$ with $\Sigma \in \mathbb{R}^{n \times n}$ a diagonal matrix of nonnegative singular values and $U_{\mathcal{T}} \in \mathbb{R}^{n \times n}$ is an orthonormal matrix of the left singular vectors of $H_{\mathcal{T}}$. The result follows by noting that $\left\|U_{\mathcal{T}}^{\top} \mathbf{x}^{*}\right\|_{2}=\left\|\mathbf{x}^{*}\right\|_{2}$ and that the nonzero entries of the diagonal matrix $\left(I-(\Sigma+\lambda I)^{-1} \Sigma\right)$ ranges from $\frac{\lambda}{\lambda+\bar{\sigma}_{\mathcal{T}}}$ to 1 .

Remark 3. As $\lambda \rightarrow 0$, the minimum estimation error approaches zero. However, the resulting Hessian matrix $H_{\mathcal{T}}^{\top} H_{\mathcal{T}}+\lambda I$ degenerates and the solution with the minimum norm have an underwhelming probability of being attained. On the other hand, as $\lambda \rightarrow \infty$, the minimum relative error approaches 100\%. However, the Hessian becomes more nonsingular in this case. This shows the trade-off involved in deciding a value for the regularization parameter.

Suppose, in addition to $\mathcal{T}$, we have a prior distribution $\operatorname{prob}\left(\mathbf{y}_{\mathcal{T}^{c}} \mid \mathbf{z}\right)$ on the unavailable measurements due to extreme ACEs. The prior information depends on the auxiliary variable $\mathbf{z}$, and is given by the GPR model obtained in previous section. We define an enhanced decoder as the minimizer of the following optimization problem: 


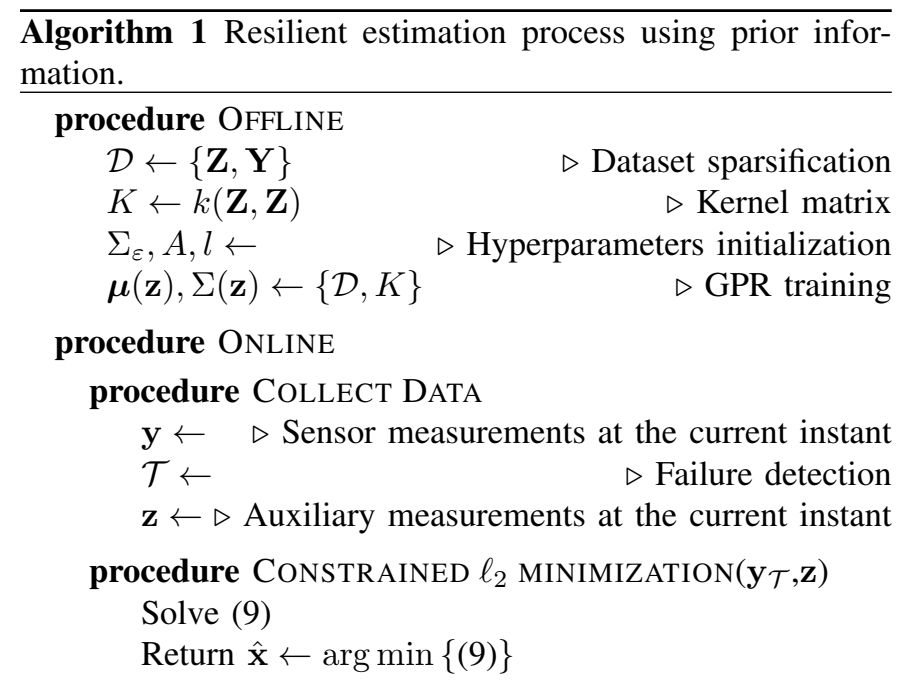

$$
\begin{array}{ll}
\text { Minimize: } & \left\|\mathbf{y}_{\mathcal{T}}-H_{\mathcal{T}} \mathbf{x}\right\|_{2}^{2}+\lambda\|\mathbf{x}\|_{2}^{2} \\
\text { Subject to: } & \\
& \|H \mathbf{x}-\boldsymbol{\mu}(\mathbf{z})\|_{\Sigma^{-1}(\mathbf{z})}^{2} \leq \chi_{m}^{2}(\tau)+\frac{\delta^{2}}{\underline{\sigma}(\mathbf{z})},
\end{array}
$$

where $\tau \in(0,1)$ is a prior reliability parameter, $\chi_{\mathcal{T}^{c}}^{2}(\tau)$ is the quantile function for probability $\tau$ of the chi-squared distribution with $m$ degrees of freedom,and $\underline{\sigma}(\mathbf{z})$ is the smallest singular value of the prior covariance matrix $\Sigma(\mathbf{z})$.

The algorithm for the resilient estimation approach of the CPES using prior information is outlined in Algorithm 1 . The solution of the constrained minimization problem in 9 is solved while considering all the necessary initialization offline steps, as well as the sensing data, auxiliary variables, and failure nodes in the system.

It is also possible to consider a different norm objective in 9). For example, a mixed $\ell_{1}$ and $\ell_{2}$ norms $\left(\left\|\mathbf{y}_{\mathcal{T}}-H_{\mathcal{T}} \mathbf{x}\right\|_{1}+\right.$ $\lambda\|\mathbf{x}\|_{2}^{2}$ ), or an pure $\ell_{1}$ norm $\left(\left\|\mathbf{y}_{\mathcal{T}}-H_{\mathcal{T}} \mathbf{x}\right\|_{1}\right)$ as widely used in resilient estimation under false data injection attack situation [22]. While such objectives have been shown to be effective in recovering the true states under adversarial situation, recoverability often requires the system matrix $H$ to satisfy some RIP conditions which are known to be very hard to verify. Moreover, since the situation considered in this paper involves complete node failures, such objective may not be as effective. This turns out to be the case as shown in the subsequent simulation results. Also, one of the main motivations for the choice of the $\ell_{2}$ objective is for uniformity with the regularization term as well as to facilitate easier comparison with least squares based state reconstruction method that is prevalent in the industry. As seen in subsequent simulations, this allows us to isolate the improvement due to the auxiliary information and to quantify the magnitude of such improvement as a function of the quality of the prior information.

The next result shows the estimation error bound for the decoder, using the prior information.
Theorem 1. Consider the decoder given by the optimization problem in (9). Suppose the true measurement satisfies the distribution $\mathbf{y}^{*} \mid \mathbf{z} \sim \mathcal{N}(\boldsymbol{\mu}(\mathbf{z}), \Sigma(\mathbf{z}))$. Then, with a probability of at least $\tau$, the decoder,given by the optimization problem in (9) satisfies the error bound below:

$$
\left\|\hat{\mathbf{x}}-\mathbf{x}^{*}\right\|_{2} \leq \sqrt{\frac{2\left(\delta^{2}+\lambda\left\|\mathbf{x}^{*}\right\|_{2}^{2}\right)}{\underline{\sigma}_{\mathcal{T}}^{2}+\lambda}},
$$

where $\underline{\sigma}_{\mathcal{T}}$ is the smallest singular value of $H_{\mathcal{T}}$ and $\hat{\mathbf{x}}, \mathbf{x}^{*}$ are the estimated and true states respectively.

Proof. Since $\mathbf{y}^{*} \mid \mathbf{z} \sim \mathcal{N}(\boldsymbol{\mu}(\mathbf{z}), \Sigma(\mathbf{z}))$, then

$$
\operatorname{prob}\left(\|\mathbf{y}-\boldsymbol{\mu}(\mathbf{z})\|_{\Sigma^{-1}(\mathbf{z})}^{2} \leq \chi_{\tau}^{2}(\tau)\right) \geq \tau \text {. }
$$

Thus, with a probability of at least $\tau$, the following holds:

$$
\left\|H \mathbf{x}^{*}+\boldsymbol{\varepsilon}-\boldsymbol{\mu}(\mathbf{z})\right\|_{\Sigma^{-1}(\mathbf{z})}^{2} \leq \chi_{\tau}^{2}(\tau),
$$

which after using the left-hand-side triangular inequality yields

$$
\begin{aligned}
\left\|H \mathbf{x}^{*}-\boldsymbol{\mu}(\mathbf{z})\right\|_{\Sigma^{-1}(\mathbf{z})}^{2} & \leq \chi_{\tau}^{2}(\tau)+\|\varepsilon\|_{\Sigma^{-1}(\mathbf{z})}^{2} \\
& \leq \chi_{\tau}^{2}(\tau)+\frac{\delta^{2}}{\underline{\sigma}(\mathbf{z})} .
\end{aligned}
$$

Thus, with a probability of at least $\tau$, the true state $\mathbf{x}^{*}$ is a feasible point of the optimization problem in (9). Consequently, from the optimality of the estimate $\hat{\mathbf{x}}$, it follows that:

$$
\begin{aligned}
\left\|\mathbf{y}_{\mathcal{T}}-H_{\mathcal{T}} \hat{\mathbf{x}}\right\|_{2}^{2}+\lambda\|\hat{\mathbf{x}}\|_{2}^{2} & \leq\left\|\mathbf{y}_{\mathcal{T}}-H_{\mathcal{T}} \mathbf{x}^{*}\right\|_{2}^{2}+\lambda\left\|\mathbf{x}^{*}\right\|_{2}^{2} \\
& \leq \delta^{2}+\lambda\left\|\mathbf{x}^{*}\right\|_{2}^{2} \\
\left\|H_{\mathcal{T}}\left(\hat{\mathbf{x}}-\mathbf{x}^{*}\right)-\varepsilon_{\mathcal{T}}\right\|_{2}^{2}+\lambda\|\hat{\mathbf{x}}\|_{2}^{2} & \leq \delta^{2}+\lambda\left\|\mathbf{x}^{*}\right\|_{2}^{2}
\end{aligned}
$$

which after using the left-hand-side triangular inequality yields

$$
\begin{aligned}
\left\|H_{\mathcal{T}}\left(\hat{\mathbf{x}}-\mathbf{x}^{*}\right)\right\|_{2}^{2}+\lambda\left\|\left(\hat{\mathbf{x}}-\mathbf{x}^{*}\right)+\mathbf{x}^{*}\right\|_{2}^{2} & \leq 2 \delta^{2}+\lambda\left\|\mathbf{x}^{*}\right\|_{2}^{2}, \\
\left(\hat{\mathbf{x}}-\mathbf{x}^{*}\right)^{\top}\left(H_{\mathcal{T}}^{\top} H_{\mathcal{T}}+\lambda I\right)\left(\hat{\mathbf{x}}-\mathbf{x}^{*}\right) & \leq 2\left(\delta^{2}+\lambda\left\|\mathbf{x}^{*}\right\|_{2}^{2}\right), \\
\text { which implies that }\left\|\hat{\mathbf{x}}-\mathbf{x}^{*}\right\|_{2}^{2} & \leq \frac{2\left(\delta^{2}+\lambda\left\|\mathbf{x}^{*}\right\|_{2}^{2}\right)}{\underline{\sigma}_{\mathcal{T}}^{2}+\lambda} .
\end{aligned}
$$

Remark 4. If the measurement noise upper bound is modeled as a fraction of the true state (i.e $\|\varepsilon\|_{2} \leq \delta\left\|\mathrm{x}^{*}\right\|_{2}$ ), then the estimation error bound becomes

$$
\frac{\left\|\hat{\mathbf{x}}-\mathbf{x}^{*}\right\|_{2}}{\left\|\mathbf{x}^{*}\right\|_{2}} \leq \sqrt{\frac{2\left(\delta^{2}+\lambda\right)}{\underline{\sigma}_{\mathcal{T}}^{2}+\lambda}} .
$$

Thus, if $\delta \ll \underline{\sigma}_{\mathcal{T}}$ and $\lambda \ll \underline{\sigma}_{\mathcal{T}}$ then

$$
\frac{\left\|\hat{\mathbf{x}}-\mathbf{x}^{*}\right\|_{2}}{\left\|\mathbf{x}^{*}\right\|_{2}}=\mathcal{O}(\delta+\lambda) \text {. }
$$

In other words, if the noise is small, the relative estimation error can be made of the order $\mathcal{O}(\delta)$ by appropriate choice of the regularization parameter $\lambda$. Moreover, as $\lambda \rightarrow 0$, the risk of degeneracy is avoided in this case thanks to the quadratic constraints due the prior model. 


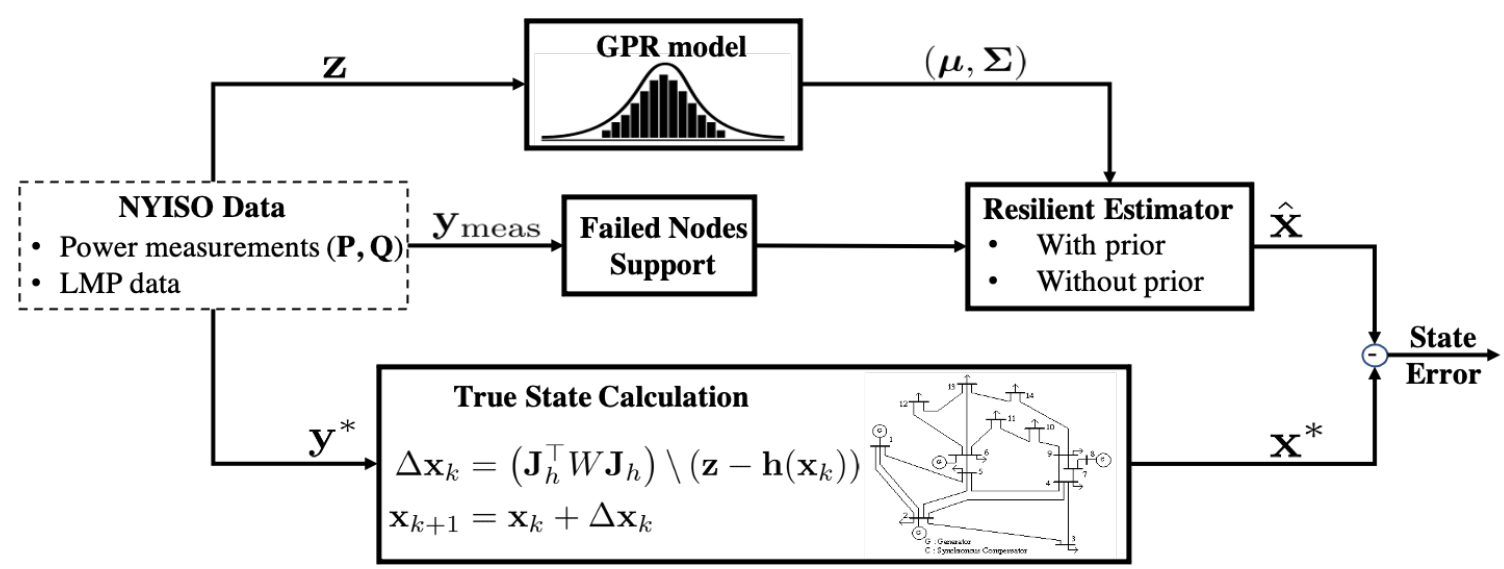

Fig. 4: Conceptual diagram of the simulation process of the CPES resilient estimation process.

\section{Simulation RESUlTS}

In order to validate the methodology of the previous sections, multiple simulation scenarios are carried out using actual load and market data from the NYISO for 3 months (between January and March) in 2017 and 2018. The conceptual diagram of the simulation process and setup is shown in Fig. 4. The real-time ( 5 minutes interval) LMP data is fed into the designed GPR model to produce an estimated mean and covariance matrix of the corresponding sensing measurements $\mathbf{y} \in \mathbb{R}^{m}$, i.e., real $(\mathbf{P})$ and reactive $(\mathbf{Q})$ power measurements. In addition, the actual power measurements are fed directly to a true state calculation scheme which gives the true states of the power system for comparison. Moreover, the true power measurements are a fed through a failed node support generator which emulates the occurrence of ACEs and where a portion of the measurements are declared unsafe and removed from the system measurement vector. The safe subset is what is passed to the resilient estimators. As mentioned within the paper, resiliency refers to the tendency to maintain low estimation error when portions of the system measurement is unavailable due to ACEs. In the simulation, this is shown by plots of the percentage successful estimation (or estimation error distribution) Vs. number of failed sensors. The state estimation errors for each estimation schemes are computed by subtracting the resulting estimates from the true states.

Two simulation scenarios are considered. The first is a failure sweep case in which different combinations for a given number of failed nodes are generated. In other words, assuming the existence of an ACE (or multiple ACEs) in a specific area of the CPES (e.g., a hurricane in a disaster-prone region) and the resulting outage of a number of nodes, we assume combinations of those failed sensing nodes. In the second scenario, the system is sequentially iterated through time and random node failures are injected at each time. The next subsections give a more detailed description of each scenario and the results obtained thereof.

\section{A. Failure Sweep Scenario}

For this simulation scenario, the number of failed nodes is swept from $23 \%$ to $100 \%$. For each instance, 1000 different random combinations of failed nodes are generated. For each combination at each instance, three estimation schemes are implemented. First, traditional robust/resilient estimation scheme is implemented by solving an unconstrained $\ell_{1}$ optimization problem to recover the states using the healthy subset of the system measurements. The second scheme implements the unconstrained least squares regression approach that is prevalent in the industry at the moment [33]. The third scheme is the proposed resilient estimator which solves a constrained least squares regression problem in Eq. (9). The corresponding quadratic constraint is generated from the GPR model developed in previous sections.

The results are shown in Fig. 5. The horizontal axis is the total number of failed measurements swept from $23 \%$ to $100 \%$, while the vertical axis is the percentage (\%) of the 1000 cases for which the corresponding estimators is successful. Success in this case is determined by comparing the estimation error with the estimator error of a typical least routine without any failed nodes. If the error is less than that of a typical least routine, the scheme is declared successful for that case. As seen from the figure, as observability dwindles due to increasing number of failed nodes, the performance of the estimators, assessed by the percentage successful estimation, reduces as expected. The results show that the proposed resilient estimators using prior information significantly outperforms both the traditional and state-of-the art schemes, with $100 \%$ successful state recovery for up to $60 \%$ node failures.

In order to further assess how the prior information affects the performance of the resulting CPES estimation scheme, additional simulation experiments are carried out by injecting biases in the GPR mean estimates. The size of the injected biases is varied to represent prior information of varying accuracy. The result is shown in Fig. 6 and Fig. 7. In the figures, the black arrow indicates the direction of increasing mean bias. As seen in Fig. 6, the resulting estimation success rates reduce with increasing bias, showing that the accuracy of the proposed estimation scheme depends on the accuracy of the prior information. This further corroborates the theoretic result given in Theorem 1 and Remark 4 The corresponding relative mean error is also shown in Fig. 7 to increase with increasing mean bias. 


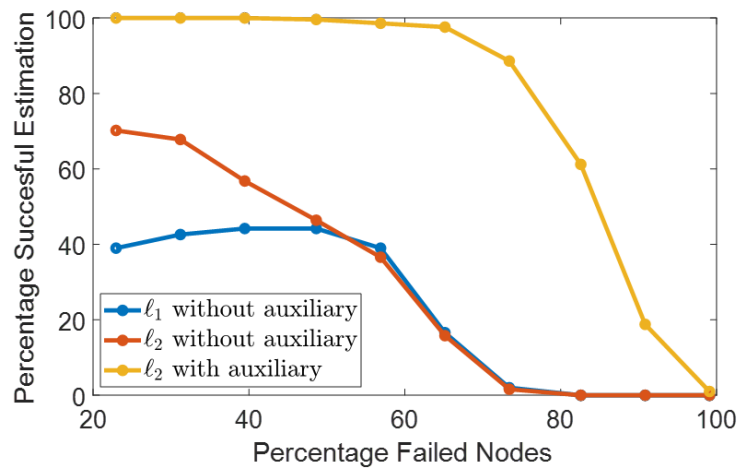

Fig. 5: Failure sweep scenario: CPES estimation success rates.

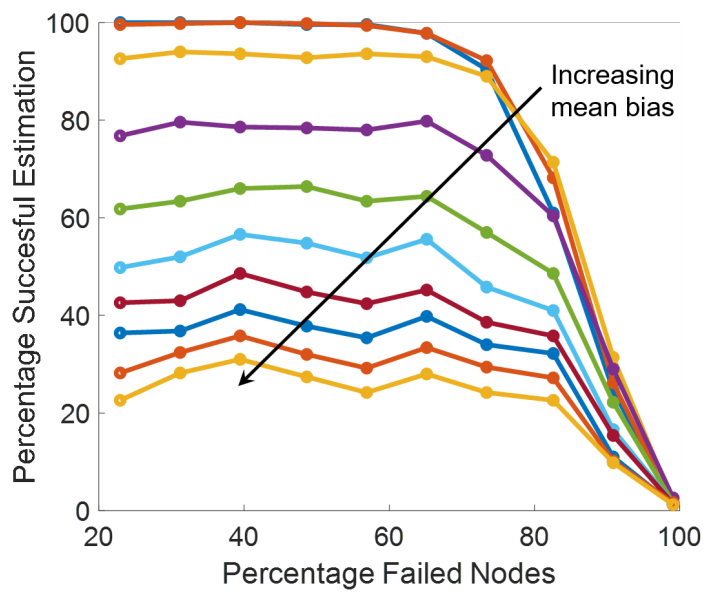

Fig. 6: Failure sweep scenario: effect of mean bias on CPES estimation success rate.

\section{B. Sequential Simulation}

For this simulation scenario, the 5-minute load data of NYISO are used to validate the performance of the proposed CPES resilient estimation under varying degree of node failures, i.e., sensing nodes are randomly affected by any ACEs, making them unresponsive in providing system measurements. The natural time history of the CPES is generated by simulating the underlying dynamical system, howbeit with random node failures injected into the system measurements at each time instant. At each time step, a random integer, $\mathrm{nFails}$, in the interval $[0$, round $(0.6 \times$ total number of measurement $)]$ is generated as the total number of node failures at the instant. Next, the random permutation of $\mathrm{nFails}$ channels is selected for failure injection. After removing the failed nodes from the measurements, resilient estimation schemes are invoked on the remaining measurement to infer the system states. For this scenario, we only considered the least square estimators, one with auxiliary constraint and the other without. The reason is because the fundamental performance difference between the various schemes has already been established in the previous scenario. In the present scenario, we are mainly interested in studying the effect of the auxiliary constraint on time-varying random node failures; that is why it is more appropriate to

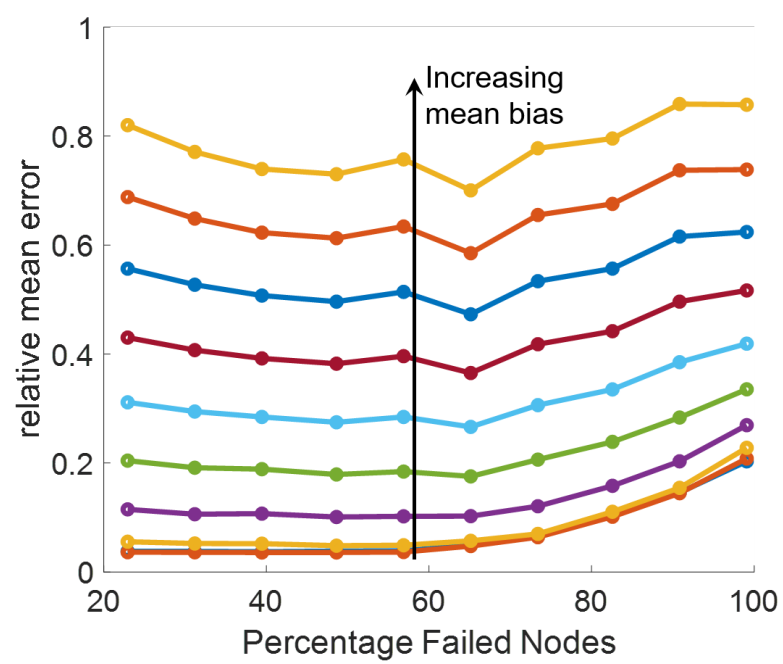

Fig. 7: Failure sweep scenario: effect of mean bias on CPES estimation relative error.

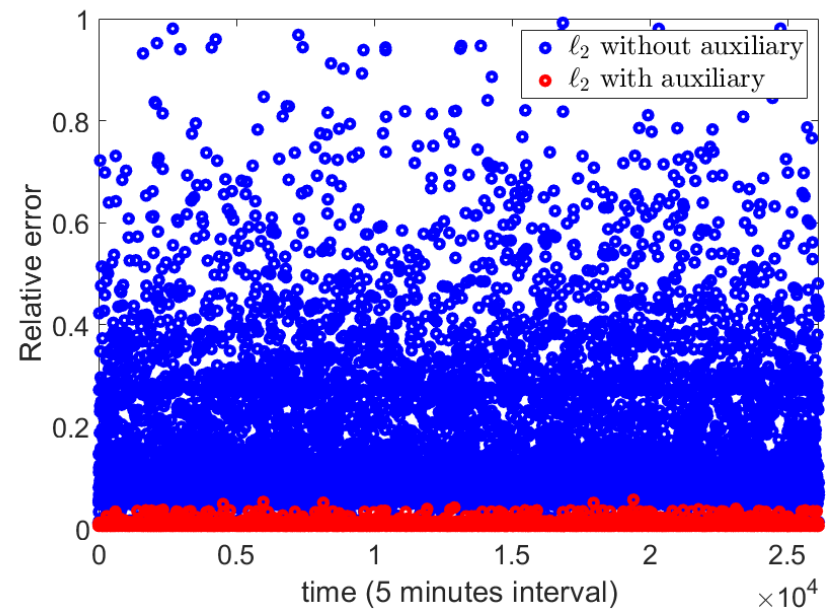

Fig. 8: Sequential simulation scenario: relative error.

compare only the least-squares-based approaches.

The results from this sequential simulation scenario are shown in Fig. 8 and Fig. 9 In, Fig. 8, the corresponding relative error is shown for each estimators. It is plotted for each time instant at 5 mins. interval. It is seen that the estimator constrained by the auxiliary information outperforms the counterpart by a significant margin. The ratio of relative errors of without auxiliary to with auxiliary ranges in the interval $[1.02,111.77]$, with a mean value of 6.58 . Even though some failure combinations resulted in a relative error of almost 1 in the unconstrained least squares case, the maximum relative error recorded for the proposed estimator is 0.06 .

In order to further understand the distribution of the relative errors, Fig. 9 shows corresponding histograms truncated at 0.3 to provide good visibility. As seen from the figure, the relative errors for the constrained estimator is concentrated near the origin, while the unconstrained estimator has significant spread far away from the origin. 

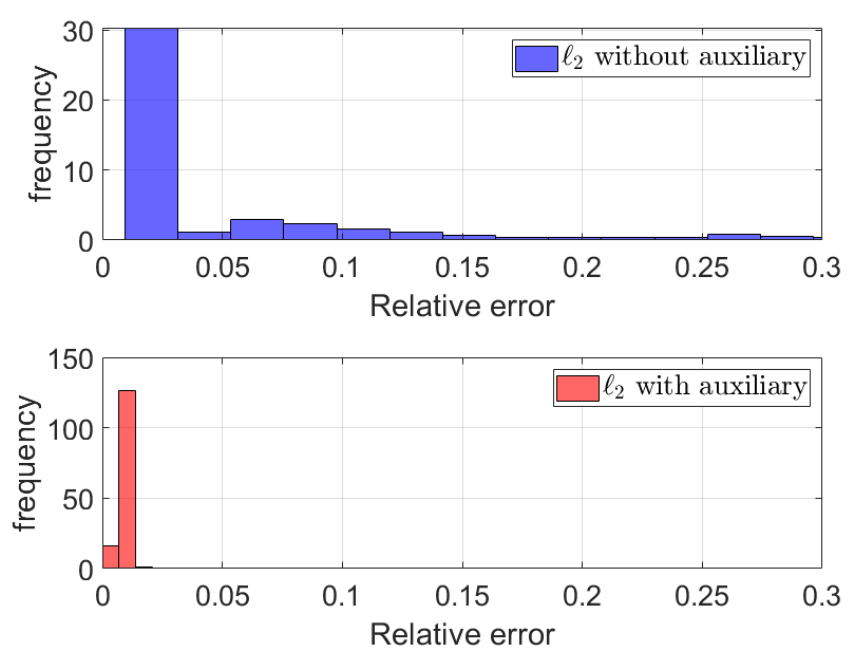

Fig. 9: Sequential simulation scenario: relative error distribution.

\section{CONCLUSION AND FUTURE WORK}

In this paper, we show that incorporation of prior measurement information can significantly improve the resiliency of optimal estimation algorithms used in CPS applications. In particular, we proved that certain prior set inclusion constraints results in much stronger reconstruction error bound. The problem is formulated as a constrained optimization problem and standard results are extended to prove the main results. In addition, numerical simulations are used to validate the theoretical claims. We tested our approach on the IEEE 14-bus system mapped to actual NYISO load data. We demonstrate that by corroborating the state estimation with prior information of the CPES, it is possible to make it much more difficult for ACEs (able to corrupt portion of CPS sensor measurements) to affect the operation of such critical infrastructure systems. Our future work will aim to extend the theoretical and algorithmic developments in this paper to the dynamic case using multiple event-triggered auxiliary models and apply the results to CPS supporting distributed resilient state estimators and moving horizon estimators. In addition, we aim to extend our work to the nonlinear case via infinite-dimensional compressed sensing in Banach space.

\section{APPENDIX - NOTATION}

The following notions and conventions are employed throughout the paper: $\mathbb{N}$ denotes the set of natural numbers. $\mathbb{R}, \mathbb{R}^{m}, \mathbb{R}^{m \times n}$ denote the space of real numbers, real vectors of length $m$ and real matrices of $m$ rows and $n$ columns respectively. $\mathbb{R}_{+}$denotes positive real numbers. $X^{\top}$ denotes the transpose of the quantity $X$. Normal-face lower-case letters $(x \in \mathbb{R})$ are used to represent real scalars, bold-face lower-case letter $\left(\mathbf{x} \in \mathbb{R}^{m}\right)$ represents vectors, normal-face upper case $\left(X \in \mathbb{R}^{m \times n}\right)$ represents matrices, while calligraphic upper case letters (e.g $\mathcal{T})$ represent sets. Let $\mathcal{T} \subseteq\{1, \ldots, m\}$ then, for a matrix $X \in \mathbb{R}^{m \times n}, X_{\mathcal{T}} \in \mathbb{R}^{|\mathcal{T}| \times n}$ and $X^{\mathcal{T}} \in \mathbb{R}^{m \times|\mathcal{T}|}$ are the sub-matrices obtained by extracting the rows, and columns respectively, of $X$ corresponding to the indices in $\mathcal{T} . \underline{\sigma}(X)$ denote the smallest singular value of the matrix $X$ respectively. For a vector $\mathbf{x}, \mathbf{x}_{i}$ denotes its $i$ th element. The support of a vector $\mathbf{x} \in \mathbb{R}^{m}$ is denoted by $\operatorname{supp}(\mathbf{x}) \triangleq$ $\left\{i: \mathbf{x}_{i} \neq 0\right\}$, with $|\operatorname{supp}(\mathbf{x})| \leq m$ being the number of nonzero elements of $\mathbf{x}$. The $p$-norm of a vector $\mathbf{x} \in \mathbb{R}^{m}$ is defined as $\|\mathbf{x}\|_{p} \triangleq\left(\sum_{i=1}^{m}\left|\mathbf{x}_{i}\right|^{p}\right)^{\frac{1}{p}}$.

\section{REFERENCES}

[1] R. Baheti and H. Gill, "Cyber-physical systems," The impact of control technology, vol. 12, no. 1, pp. 161-166, 2011.

[2] T. Sanislav and L. Miclea, "Cyber-physical systems-concept, challenges and research areas," Journal of Control Engineering and Applied Informatics, vol. 14, no. 2, pp. 28-33, 2012.

[3] I. Zografopoulos, J. Ospina, X. Liu, and C. Konstantinou, "Cyberphysical energy systems security: Threat modeling, risk assessment, resources, metrics, and case studies," IEEE Access, vol. 9, pp. 2977529818,2021

[4] C. Konstantinou, G. Stergiopoulos, M. Parvania, and P. EstevesVerissimo, "Chaos engineering for enhanced resilience of cyber-physical systems," arXiv preprint arXiv:2106.14962, 2021.

[5] S. Ntalampiras, "Automatic identification of integrity attacks in cyberphysical systems," Expert Systems with Applications, vol. 58, pp. 164 $173,2016$.

[6] V. Venkatasubramanian, R. Rengaswamy, K. Yin, and S. N. Kavuri, "A review of process fault detection and diagnosis: Part i: Quantitative model-based methods," Computers \& Chemical Engineering, vol. 27, no. 3, pp. $293-311,2003$.

[7] Z. Huang, Y. Chen, and D. Chavarría-Miranda, "High-performance computing for real-time grid analysis and operation," in High performance computing in power and energy systems. Springer, 2013, pp. 151-188.

[8] X. Liu, Z. Li, and Z. Li, "Impacts of bad data on the PMU based line outage detection," arXiv preprint arXiv:1502.04236, 2015.

[9] U.S. Department of Energy, "Strengthening the Security and Resilience of the Nation's Critical Energy Infrastructure," [Online]. Available: https://www.energy.gov/oe/articles/strengthening-securityand-resilience-nation-s-critical-energy-infrastructure 2018.

[10] A. A. Cárdenas, S. Amin, and S. Sastry, "Research challenges for the security of control systems." in HotSec, 2008.

[11] M. Pajic, P. Tabuada, I. Lee, and G. J. Pappas, "Attack-resilient state estimation in the presence of noise," in 2015 54th IEEE Conference on Decision and Control (CDC). IEEE, 2015, pp. 5827-5832.

[12] S. Z. Yong, M. Zhu, and E. Frazzoli, "Resilient state estimation against switching attacks on stochastic cyber-physical systems," in 2015 54th IEEE Conference on Decision and Control (CDC). IEEE, 2015, pp. 5162-5169.

[13] Q. Hu, D. Fooladivanda, Y. H. Chang, and C. J. Tomlin, "Secure state estimation for nonlinear power systems under cyber attacks," in 2017 American Control Conference (ACC). IEEE, 2017, pp. 2779-2784.

[14] M. Pajic, J. Weimer, N. Bezzo, P. Tabuada, O. Sokolsky, I. Lee, and G. J. Pappas, "Robustness of attack-resilient state estimators," in ICCPS'14: ACM/IEEE 5th International Conference on Cyber-Physical Systems (with CPS Week 2014). IEEE, 2014, pp. 163-174.

[15] V. Kekatos and G. B. Giannakis, "Distributed robust power system state estimation," IEEE Transactions on Power Systems, vol. 28, no. 2, pp. 1617-1626, 2012.

[16] R. Deng, G. Xiao, R. Lu, H. Liang, and A. V. Vasilakos, "False data injection on state estimation in power systems-attacks, impacts, and defense: A survey," IEEE Transactions on Industrial Informatics, vol. 13, no. 2, pp. 411-423, 2016

[17] G. Fiore, Y. H. Chang, Q. Hu, M. D. Di Benedetto, and C. J. Tomlin, "Secure state estimation for cyber physical systems with sparse malicious packet drops," in 2017 American Control Conference (ACC). IEEE, 2017, pp. 1898-1903.

[18] L. K. Mestha, O. M. Anubi, and M. Abbaszadeh, "Cyber-attack detection and accommodation algorithm for energy delivery systems," in Control Technology and Applications (CCTA), 2017 IEEE Conference on. IEEE, 2017, pp. 1326-1331.

[19] Y. Zheng and O. M. Anubi, "Attack-resilient weighted $\ell_{1}$ observer with prior pruning," arXiv preprint arXiv:2104.03580, 2021. 
[20] T. Shinohara, T. Namerikawa, and Z. Qu, "Resilient reinforcement in secure state estimation against sensor attacks with a priori information," IEEE Transactions on Automatic Control, vol. 64, no. 12, pp. 50245038, 2019.

[21] O. M. Anubi, L. Mestha, and H. Achanta, "Robust resilient signal reconstruction under adversarial attacks," arXiv:1807.08004, 2018.

[22] O. M. Anubi and C. Konstantinou, "Enhanced resilient state estimation using data-driven auxiliary models," IEEE Transactions on Industrial Informatics, vol. 16, no. 1, pp. 639-647, 2020.

[23] H. Fawzi, P. Tabuada, and S. Diggavi, "Secure estimation and control for cyber-physical systems under adversarial attacks," IEEE Transactions on Automatic Control, vol. 59, no. 6, pp. 1454-1467, 2014.

[24] Y. H. Chang, Q. Hu, and C. J. Tomlin, "Secure estimation based kalman filter for cyber-physical systems against sensor attacks," Automatica, vol. 95 , pp. 399-412, 2018.

[25] E. J. Candes and T. Tao, "Decoding by linear programming," IEEE transactions on information theory, vol. 51, no. 12, pp. 4203-4215, 2005.

[26] NYISO. Load data. [Online]. Available: http://www.nyiso.com/public/ markets_operations/market_data/load_data/index.jsp

[27] C. Konstantinou and M. Maniatakos, "A case study on implementing false data injection attacks against nonlinear state estimation," in Proceedings of the 2nd ACM Workshop on Cyber-Physical Systems Security and Privacy. ACM, 2016, pp. 81-92.

[28] C. Konstantinou, M. Sazos, A. S. Musleh, A. Keliris, A. Al-Durra, and M. Maniatakos, "Gps spoofing effect on phase angle monitoring and control in a real-time digital simulator-based hardware-in-the-loop environment," IET Cyber-Physical Systems: Theory \& Applications, vol. 2, no. 4, pp. 180-187, 2017.

[29] I. Zografopoulos, C. Konstantinou, N. G. Tsoutsos, D. Zhu, and R. Broadwater, "Security assessment and impact analysis of cyberattacks in integrated T\&D power systems," arXiv preprint arXiv:2102.03215, 2021.

[30] Z. Zhang, R. Deng, D. K. Yau, P. Cheng, and J. Chen, "Analysis of moving target defense against false data injection attacks on power grid," IEEE Transactions on Information Forensics and Security, vol. 15, pp. 2320-2335, 2019

[31] L. Liu and A. Zobian, "The importance of marginal loss pricing in an rto environment," The Electricity Journal, vol. 15, no. 8, pp. 40-45, 2002.

[32] M. Liu, G. Chowdhary, B. C. Da Silva, S.-Y. Liu, and J. P. How, "Gaussian processes for learning and control: A tutorial with examples," IEEE Control Systems Magazine, vol. 38, no. 5, pp. 53-86, 2018.

[33] X. He, Z. Wang, Y. Liu, and D.-H. Zhou, "Least-squares fault detection and diagnosis for networked sensing systems using a direct state estimation approach," IEEE Transactions on Industrial Informatics, vol. 9 , no. 3, pp. 1670-1679, 2013.

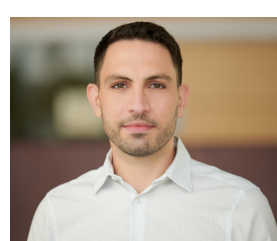

Charalambos Konstantinou (S'11-M'18-SM'20) is an Assistant Professor of Computer Science (CS) and Affiliate Professor of Electrical and Computer Engineering (ECE) at the Computer, Electrical and Mathematical Sciences and Engineering Division (CEMSE) of King Abdullah University of Science and Technology (KAUST), Thuwal, Saudi Arabia. He is the Principal Investigator of the Secure Next Generation Resilient Systems Lab (SENTRY - sentry.kaust.edu.sa and a member of the Resilient Computing and Cybersecurity Center (RC3 -

rc3.kaust.edu.sa) at KAUST. His research interests are in secure, trustworthy, and resilient cyber-physical and embedded IoT systems. He is also interested in critical infrastructures security and resilience with special focus on smart grid technologies, renewable energy integration, and real-time simulation. $\mathrm{He}$ received a Ph.D. in Electrical Engineering from New York University (NYU), NY, in 2018, and a Dipl.-Ing.-M.Eng. Degree in Electrical and Computer Engineering from National Technical University of Athens (NTUA), Greece, in 2012. Before joining KAUST, he was an Assistant Professor with the Center for Advanced Power Systems (CAPS) at Florida State University (FSU). Konstantinou has authored multiple articles in the IEEE/ACM transactions and conference proceedings and serves in the program committee of several international conferences. He is currently the Chair of the IEEE Task Force on Resilient and Secure Large-Scale Energy Internet Systems and the Secretary of the IEEE Task Force on Cyber-Physical Interdependence for Power System Operation and Control. He is the recipient of the 2020 Myron Zucker Student-Faculty Grant Award from IEEE Foundation, the Southeastern Center for Electrical Engineering Education (SCEEE) Young Faculty Development Award 2019, and the best paper award at the International Conference on Very Large Scale Integration (VLSI-SoC) 2018. He serves as the (Guest) Associate Editor for the International Journal of Electrical Power \& Energy Systems, IEEE Computer, and IEEE Consumers Electronics Magazine. He is a Senior Member of IEEE, a member of ACM, and an ACM Distinguished Speaker (2021-2024).

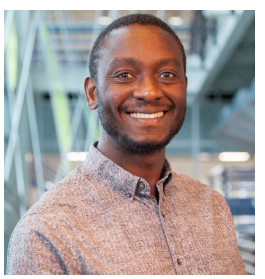

Olugbenga Moses Anubi (Senior Member, IEEE) is an Assistant Professor in the Electrical and Computer Engineering Department at the Florida State University (FSU), with affiliations with the Center for Advanced Power Systems (CAPS) and the Center for Intelligent Systems, Controls and Robotics (CISCOR). He received a Ph.D in Mechanical Engineering, from the University of Florida. Prior to joining FSU, he was a Lead Control Systems Engineer at GE Global Research, NY. His work within GE resulted in 12 Patent Applications and several recognitions including the GE Technology Award (Physical+Digital), the Connected Controls Technical Achievement Award, the Whitney Award and the Dushman Technology Award. His research interests include robust, resilient and adaptive control systems, real-time optimization, robotics, and vehicle dynamics \& control. 